\title{
A Missense Mutation in OPA1 Causes Dominant Optic Atrophy in a Chinese Family
}

\author{
Shaoyi Mei $\mathbb{D}$, ${ }^{1}$ Xiaosheng Huang $\mathbb{D},{ }^{1,2}$ Lin Cheng $\mathbb{D},{ }^{3}$ Shiming Peng $\mathbb{D},{ }^{1,2}$ Tianhui Zhu $\mathbb{D},{ }^{1,2}$ \\ Liang Chen $\mathbb{D}^{1},{ }^{1}$ Yan Wang $\mathbb{D}^{1},{ }^{1}$ and Jun Zhao $\mathbb{D}^{1,2}$ \\ ${ }^{1}$ Shenzhen Eye Hospital Affiliated to Jinan University, Shenzhen Eye Institute, Shenzhen, Guangdong 518040, China \\ ${ }^{2}$ School of Optometry Affiliated to Shenzhen University, Shenzhen, Guangdong 518040, China \\ ${ }^{3}$ State Key Laboratory of Ophthalmology, Zhongshan Ophthalmic Center, Sun Yat-sen University, Guangzhou 510060, China
}

Correspondence should be addressed to Jun Zhao; doctorzhaojun@163.com

Received 25 January 2019; Revised 23 July 2019; Accepted 13 September 2019; Published 3 November 2019

Academic Editor: Arturo Carta

Copyright (c) 2019 Shaoyi Mei et al. This is an open access article distributed under the Creative Commons Attribution License, which permits unrestricted use, distribution, and reproduction in any medium, provided the original work is properly cited.

Background. To investigate the genetic causes and clinical characteristics of dominant optic atrophy (DOA) in a Chinese family. Methods. A 5-generation pedigree of 35 family members including 12 individuals affected with DOA was recruited from Shenzhen Eye Hospital, China. Four affected family members and one unaffected family member were selected for whole exome sequencing. Sanger sequencing was used to confirm and screen the identified mutation in 18 members of the family. The disease-causing mutation was identified by bioinformatics analysis and confirmed by segregation analysis. The clinical characteristics of the family members were analyzed. Results. A heterozygous missense mutation (c.1313A $>$ G, p.D438G) in optic atrophy 1 (OPA1) was identified in 10 individuals affected with DOA in this family. None of the unaffected family members had the mutation. Patients in this family had vision loss since they were children or adolescence. The visual acuity decreased progressively to hand movement, except for one patient (IV-12) who had relatively good vision of 20/30 and 20/28. The fundus typically manifested as optic disc pallor. The visual fields, optical coherence tomography, and visual evoked potential suggested variable degree of abnormality in patients. Patients who had a history of cigarette smoking and alcohol drinking had more severe clinical manifestations. Conclusions. Our results suggest that the p.D438G mutation in OPA1 causes optic atrophy in this family. The patients who carried the mutation demonstrated heterogeneous clinical manifestations in this family. This is the first report on the c.1313A>G (p.D438G) mutation of OPA1 in a Chinese family affected with DOA.

\section{Introduction}

Optic atrophy is an irreversible vision loss disease due to primary death of retinal ganglion cell (RGC) and its axons. Dominant optic atrophy (DOA), also known as Kjer's optic neuropathy [1], is regarded as a representative of one of the two classic paradigms of mitochondrial dysfunction in inherited optic neuropathies, with Leber's hereditary optic neuropathy (LHON) being the other [2]. The incidence of DOA is estimated to be $1: 50,000$ with prevalence as high as $1: 10,000$ in the Danish population [3]. Although the disease has a high penetrance (88\%), severity and progression of DOA are extremely variable even within the same family [4].
DOA has an insidious onset, and visual loss usually occurs in the first two decades of life [5]. The disease is characterized by a bilateral degeneration of optic nerves, primarily affecting the RGCs and their axons forming the optic nerve. The patients suffer moderate-to-severe visual loss, associated with central or paracentral visual field deficits and color vision defects. Ophthalmic examinations disclose on fundoscopy optic disc pallor or atrophy, related to the RGC death [6]. Some DOA patients harbor extraocular multisystemic features, named as dominant optic atrophy plus syndromes (DOA+), such as sensorineural deafness, or less commonly chronic progressive external ophthalmoplegia, ataxia and/or myopathy [7], or nonsyndromic, idiopathic Parkinson's disease [8]. 
DOA is mainly caused by mutations in OPA1, a nuclear gene located on chromosome $3 \mathrm{q}$, which encodes a mitochondrial dynamin-related GTPase protein that appears to be involved in mitochondrial membrane biogenesis and stabilization of membrane integrity [6,9-11]. The mutation of OPA1 will lead to mitochondrial dysfunction and subsequently apoptosis of the retinal ganglion cells. Therefore, damage of the papillomacular bundle in DOA is the consequence of the mitochondrial dysfunction caused by OPA 1 mutation [12]. DOA and LHON are difficult to be differentiated especially when the patients are in childhood, while genetic testing can assist in diagnosis of the disease [13].

In this study, we described a Chinese family affected with DOA caused by a heterozygous missense mutation in OPA1. Ten affected patients had visual loss, pale optic disc, lower amplitude and longer latency of visual evoked potential (VEP) test, thinner retinal nerve fiber layer (RNFL), and narrow visual fields.

\section{Methods}

2.1. Patients and Ophthalmological Examinations. A 5generation pedigree of 35 family members including 12 individuals affected with DOA was recruited from Shenzhen Eye Hospital, China. All of the family members are Han Chinese. DOA was diagnosed according to the following criteria [6]: (1) all patients have bilateral and symmetric moderate-to-severe progressive visual loss during their early childhood and typically occurring in the context of a family history of DOA; (2) fundoscopy reveals full pallor or temporal pallor of optic disc on both eyes; (3) central or paracentral scotoma and some patients may have extensive visual field defects (VFD); (4) optical coherence tomography (OCT) discloses nonspecific thinning of RNFL especially at the temporal part but a normal morphology of the photoreceptor layers; (5) abnormal VEP; (6) excluding optic atrophy induced by other congenital malformations from the eye or other systems and trauma; and (7) molecular genetic analysis allows patients to be adequately diagnosed.

The family members underwent the following ophthalmological examinations including best corrected visual acuity (BCVA), color testing, anterior segment exam, fundoscopy, visual field, OCT, VEP, and intraocular pressure (IOP). Optic disc, RNFL thickness, and macula were examined using OCT in 10 eyes of 5 affected patients (DOA group) and 6 eyes of 3 unaffected members (control group). The thickness of superior, inferior, temporal, and nasal quadrants of RNFL was analyzed, respectively. Visual field and VEP were measured with different strategies according to patients' vision. Those with vision above finger counting were measured by dynamic balance test strategy in visual field and pattern visual evoked potential (P-VEP). Patients who had vision of finger counting or hand movement were measured by central low vision test strategy in visual field and flash visual evoked potential (F-VEP).

The study protocol was approved by the Independent Ethics Committee of Shenzhen Eye Hospital in accordance with the tenets of the Declaration of Helsinki. Written informed consent was obtained from all study participants.
2.2. Genomic DNA Extraction and Whole Exome Sequencing. Genomic DNA was extracted from blood samples of 18 family members (10 affected individuals: III-1, III-3, III-9, III-10, IV-3, IV-5, IV-8, IV-10, IV-12, and V-2; 8 unaffected members: III-5, IV-1, IV-4, IV-6, IV-13, V-1, V-3, and V-6). The final concentration of DNA samples was diluted to $50 \mathrm{ng} / \mu \mathrm{L}$. Whole exome sequencing (WES) [14] was performed for 5 family members, including four affected individuals (i.e., V-2, III-3, III-9, and IV-5) and one unaffected member (i.e., IV-1) by the Novogene company (Beijing, China). For each sample, $0.4 \mu \mathrm{g}$ of genomic DNA was randomly sonicated into fragments and used to construct a paired-end sequencing library. Exome capture was performed using a SureSelect Human All Exon v6+UTR capture kit (Agilent Technologies, Santa Clara, CA). Each sample underwent $2 \times 150 \mathrm{bp}$ paired-end sequencing on a HiSeq 4000 Next-Generation Sequencing system (Illumina, San Diego, CA). The sequenced reads were aligned to the human reference genome (hg19) using the Burrows-Wheeler Aligner (BWA) [15]. Sequence variants were called using Samtools and GATK $[16,17]$ and annotated with ANNOVAR [18]. The transcript variant NM_015560 was used to denominate the mutation of OPA1.

\subsection{Variant Filtering and Mutation Verification.} Sequence variants were filtered based on the following criteria: (1) novel or rare in population database: minor allele frequency $<1 \%$ in Exome Aggregation Consortium (ExAC); (2) most likely pathogenic: pathogenicity score of at least "possibly damaging" or "disease causing" after annotation by Sorts Intolerant From Tolerant (SIFT), Polymorphism Phenotyping v2 (Polyphen-2), and MutationTaster; (3) evidence of evolutionary conservation (GERP score >0); (4) Combined Annotation Dependent Depletion (CADD score $>15$ ); and (5) heterozygotes consistent with the dominant inheritance pattern.

The identified mutation in OPA1 was verified using polymerase chain reaction (PCR) and Sanger sequencing. The primer sequences used in PCR and Sanger sequencing were: (1) forward primer: 5'-GCCATCATACTGTGTATT CAAGG-3' and (2) reverse primer: $5^{\prime}$-GTGAGCCTGGTT TCCTTCAG-3'.

2.4. Statistical Analysis. Statistical analysis was performed using the SPSS 24.0 software (SPSS Inc, Chicago, IL). Since the RNFL thickness in the nasal quadrant followed normal distribution, the data were presented as mean \pm standard deviation. RNFL thickness in this quadrant was compared between patients with DOA and unaffected controls using $t$ test. While the RNFL thickness in the superior, inferior, and temporal quadrants did not follow normal distribution, the data were presented as median (quartile 1 and quartile 3). RNFL thickness in three quadrants was compared between patients with DOA and unaffected controls using MannWhitney $U$ test. The BCVA was compared between smokers and nonsmokers or between alcohol drinkers and nondrinkers in patients of this family using the Student's $t$-test. The logMAR figures " 2.30 " and " 1.85 " were applied for those 
subjects tested as "HM" and "FC," respectively [19]. $P<0.05$ was considered statistically significant.

\section{Results}

3.1. DOA Pedigree and Patients. The pedigree consisted of 35 family members from 5 generations. There was no consanguineous marriage in this family. The male-to-female ratio in patients with DOA was 7:5. These pedigree features together with male-to-male transmissions suggested an autosomal dominant inheritance in this family (Figure 1). Twelve individuals in this family had vision loss (I-1, II-1, III-1, III-3, III-9, III-10, IV-3, IV-5, IV-8, IV-10, IV-12, and $\mathrm{V}-2)$, and the others had normal vision. The proband (V-2) and her mother (IV-3) had vision loss; however, the vision of her father (IV-4) was normal. V-2 was born at full term without systemic diseases and bad habits. She was diagnosed as optic atrophy because of poor vision at 3 years old. The patient III-1 had DOA accompanied with left ear hearing loss, and he had drinking history for 60 years. The patient III-3 was diagnosed with rheumatic osteoarthrosis at 30 years old and left femoral head necrosis at 58 years old, and he had smoking and drinking history for more than 35 years. Patients III-9 and III-10 are twin brothers. Patient III-9 had smoking and drinking history for more than 20 years. $\mathrm{Pa}-$ tient III-10 was diagnosed with blue and yellow color anomalopia at 20 years old, and he had smoking and drinking history for more than 15 years. Patient IV-5 was treated using intravenous mouse nerve growth factor in 2012, but it was not effective, and he had drinking history for 8 years. Patient IV-10 had smoking and drinking history for more than 15 years. All patients had clear or mild dense lens and normal macula lutea and IOP (less than $21 \mathrm{mmHg}$ ). Except that I-1 and II-1 were deceased, clinical characteristics of the other 10 patients with DOA are shown in Table 1. Analysis of the clinical characteristics of the 10 patients showed remarkable variable expressivity, and phenotypic heterogeneity were observed among patients.

3.2. Eye Manifestations. The severity of vision loss in this family varied tremendously. BCVA ranged from hand movement to $20 / 28$ (Table 1). The BCVA in smokers was significantly lower than in nonsmokers among patients of this family $(P<0.05$; Table 2$)$. The BCVA in alcohol drinkers was significantly lower than in nondrinkers among patients of this family $(P<0.001$; Table 2$)$. Fundoscopy suggested full pallor or temporal pallor of optic disc on both eyes (Figure 2). The RNFL thickness in four quadrants of the DOA group was significantly thinner than in unaffected controls by OCT $(Z=3.256, P<0.001 ; Z=3.271, P<0.001 ; Z=3.259$, $P<0.001 ; t=4.863, P<0.05$ in superior, inferior, temporal, and nasal quadrants, respectively) (Figures 3 and 4). F-VEP suggested mild-to-severe prolonged $\mathrm{P}$-wave latencies and lower P wave; P-VEP presented mild-to-severe P100 amplitude reduction (Figure 5).

3.3. Identification of the OPA1 Mutation. The WES data of 5 family members (4 patients: V-2, III-3, III-9, and IV-5 and one healthy member: IV-1) captured $99.8 \%-99.9 \%$ of all exons in the genome. The average read depth was over $114 \times$. 99.6\% targeted regions were covered for $10 \times$, and $98.4 \%$ targeted regions were covered for $20 \times$ times. A total of 392,379 single nucleotide polymorphisms (SNPs) and 58,595 indels were called from the WES data (Table 3). After variant filtering, 1,900 SNPs and 884 indels were obtained for further analysis. After checking the segregation of variants with the disease status in the family, a missense mutation (c.1313A>G, p.D438G) in the OPA1 gene located at 3q29 screened by WES was the best candidate mutation or likely responsible for the disease in this family. The mutation was predicted to be pathogenic (SIFT $=0.005$, possibly damaging; Polyphen-2 $=1.0$, possibly damaging; MutationTaster $=1.0$, disease causing; and CADD = 28.9) and was extremely conservative (GERP score: 5.8 ).

3.4. Verification of the OPA1 Mutation. The OPA1 mutation (c.1313A > G, p.D438G) was verified using PCR and Sanger sequencing in all 4 DOA patients who underwent WES. Further sequencing of the other 12 family members who had DNA samples in this pedigree confirmed segregation of the mutation with the disease status in this family (Figure 6). No mtDNA mutation in $L H O N$ was found in all family members who had DNA samples (data not shown).

\section{Discussion}

In this study, we identified an OPA1 missense mutation (c.1313A $>$ G, p.D438G) responsible for the disease in a Chinese family affected with DOA (Figure 1). The OPA1 gene is located at 3q28-29 and has 31 exons and 8 transcript variants. OPA1 encodes a mitochondrial dynamin-related GTPase, which is localized to the inner mitochondrial membrane and helps regulate mitochondrial stability and energy output. This protein is also required for maintaining the integrity of cristae junctions and preventing the leakage of cytochrome C [20-22]. The OPA1 protein shares several structural features with dynamins, including a GTPase domain containing three consensus: GTP-binding sequences and a dynamin segment, a middle domain, and a GTPase effector domain containing a coiled-coil region. Among these, the GTPase domain and the C-terminal coiled-coil domain are most common damaged areas of DOA [23].

The c.1313A $>$ G (p.D438G) mutation of OPA1 identified in this study is for the first time found in the Chinese population, although it was initially reported in an Iranian DOA family [24]. It is hypothesized that the p.D438G mutation leads to misalignment of GTP in the GTP-binding pocket of the GTPase domain of OPA1. As a consequence, GTP hydrolysis will be impaired, resulting in reduced mitochondrial function. Initially, carriers cope well with the ensuing energy deficiency [24]. However, the highly energydemanding and delicate retinal ganglion neurons eventually suffer gradual damage due to the continuous lack of sufficient energy, leading to the insidious onset of visual loss, a characteristic for optic atrophy [24]. We concur with their 


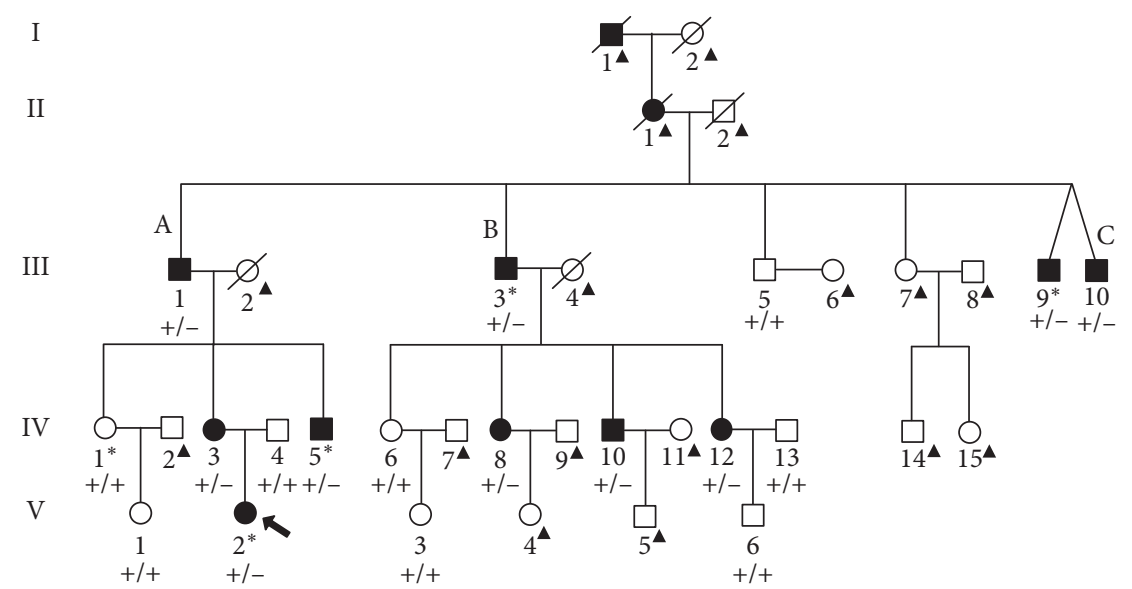

Figure 1: The pedigree structure of the DOA family. Slashes denote deceased family members. The arrow indicates the proband. Squares denote the male family members, and circles denote the female family members. Solid black symbols denote vision loss patients, and open white symbols denote unaffected members. III-9 and III-10 are twins. The number below each member denotes the tag of each patient. The letters A, B, and C above three subfamilies denote their subfamily tags. The individuals marked with an asterisk $(*)$ were analyzed by whole exome sequencing. For individuals marked with a triangle $(\mathbf{\Lambda})$, no blood sample was available. Genotypes of the OPA1 mutation (c.1313A > G, p.D438G) are indicated below each pedigree symbol (+, wildtype allele; -, mutant allele).

TABLE 1: Clinical characteristics of patients with DOA in the Chinese family.

\begin{tabular}{|c|c|c|c|c|c|c|c|c|c|c|}
\hline \multirow{2}{*}{$\begin{array}{l}\text { Subfamily } \\
\text { Patient }\end{array}$} & \multicolumn{4}{|c|}{ A } & \multicolumn{4}{|c|}{$\mathrm{B}$} & \multicolumn{2}{|c|}{$\mathrm{C}$} \\
\hline & III-1 & IV-3 & IV-5 & $\mathrm{V}-2$ & III-3 & IV-8 & IV -10 & IV-12 & III-9 & III-10 \\
\hline Gender & M & $\mathrm{F}$ & $\mathrm{M}$ & $\mathrm{F}$ & M & $\mathrm{F}$ & M & $\mathrm{F}$ & M & M \\
\hline $\begin{array}{l}\text { Age at enrollment/age at } \\
\text { diagnosis (years) }\end{array}$ & $77 / 10$ & $28 / 3$ & $23 / 9$ & $8 / 3$ & $63 / 14$ & $35 / 15$ & $33 / 18$ & $29 / 16$ & $52 / 13$ & $52 / 5$ \\
\hline Smoking/drinking & $-1+$ & $-1-$ & $-1+$ & $-1-$ & $+/+$ & $-1-$ & $+/+$ & $-1-$ & $+/+$ & $+/+$ \\
\hline BCVA(OD,OS) & $\mathrm{HM}, \mathrm{HM}$ & FC,FC & $\begin{array}{l}20 / 500 \\
20 / 500\end{array}$ & $20 / 125,20 / 125$ & $\mathrm{HM}, \mathrm{HM}$ & $\begin{array}{l}20 / 100 \\
20 / 100\end{array}$ & $\begin{array}{l}20 / 333 \\
20 / 667\end{array}$ & $20 / 30,20 / 28$ & FC,FC & HM,HM \\
\hline Pupil (mm) & $5 * 5$ & $\begin{array}{l}4.5 * 4.5 \\
\operatorname{RAPD}(+)\end{array}$ & $\begin{array}{l}4.5 * 4.5 \\
\text { RAPD }(+)\end{array}$ & Normal & $\begin{array}{l}5.5 * 5.5 \\
\text { RAPD }(+)\end{array}$ & Normal & $\begin{array}{l}3.5 * 3.5 \\
\operatorname{RAPD}(+)\end{array}$ & Normal & $\begin{array}{l}4.5 * 4.5 \\
\text { RAPD }(+)\end{array}$ & $\begin{array}{c}6 * 6 \\
\operatorname{RAPD}(+)\end{array}$ \\
\hline Optic disc & Pale & Pale & Pale & Temporal pale & Pale & Temporal pale & Pale & Temporal pale & $\begin{array}{l}\text { Pale } \\
\text { Family }\end{array}$ & $\begin{array}{c}\text { Pale } \\
\text { member }\end{array}$ \\
\hline Lab tests & $\begin{array}{c}\text { Family m } \\
\text { (OU); sev } \\
\text { member } \\
\text { (OU); ex } \\
\text { OCT ( } \\
\text { member I } \\
\text { VEP (OS) } \\
\text { VEP (OU }\end{array}$ & $\begin{array}{l}\text { ember IV-3 } \\
\text { verely abnor } \\
\text { r IV-5: mod } \\
\text { xtensively th } \\
\text { OU); exten } \\
\text {-2: mildly } \\
\text { ); moderatel } \\
\text { ); extensive } \\
\text { of O }\end{array}$ & $\begin{array}{l}\text { 3: mildly ab } \\
\text { rmal P-VEP } \\
\text { derately abn } \\
\text { hinned RNF } \\
\text { sive VFD ( } \\
\text { to moderate } \\
\text { ely to severe } \\
\text { ely thinned I } \\
\text { CT (OU). }\end{array}$ & $\begin{array}{l}\text { normal F-VEP } \\
\text { (OU). Family } \\
\text { tormal F-VEP } \\
\text { OU). Family of } \\
\text { eU abnormal F- } \\
\text { ely abnormal P- } \\
\text { RNFL thickness }\end{array}$ & $\begin{array}{r}\text { Family } \\
\text { abnorma } \\
\text { thickne } \\
\text { tempora }\end{array}$ & $\begin{array}{l}\text { y member III-3: } \\
\text { al F-VEP (OS); e } \\
\text { ss of OCT (OU) } \\
\text { a thinned RNFL } \\
\text { extensive }\end{array}$ & $\begin{array}{l}\text { moderately } \\
\text { xtensively th } \\
\text { Family me } \\
\text { thickness of } \\
\text { VFD (OU). }\end{array}$ & $\begin{array}{l}\text { to severely } \\
\text { hinned RNFL } \\
\text { ember IV-10: } \\
\text { f OCT (OU); }\end{array}$ & $\begin{array}{l}\text { Family } \\
\text { III-9: moc } \\
\text { severely } \\
\text { F-VEP } \\
\text { extensivel } \\
\text { RNFL thi } \\
\text { OCT (OU } \\
\text { VFD (OL } \\
\text { color an } \\
\text { Family m } \\
\text { 10: mod } \\
\text { severely } \\
\text { F-VEP ( } \\
\text { and yell } \\
\text { anomalo }\end{array}$ & $\begin{array}{l}\text { member } \\
\text { derately to } \\
\text { abnormal } \\
\text { (OU); } \\
\text { ly thinned } \\
\text { ickness of } \\
\text { ); extensive } \\
\text { J); yellow } \\
\text { omalopia. } \\
\text { ember III- } \\
\text { erately to } \\
\text { abnormal } \\
\text { OS); blue } \\
\text { ow color } \\
\text { pia (OU). }\end{array}$ \\
\hline
\end{tabular}

M: male; F: female; BCVA: best corrected visual acuity; OU: eye, both; OD: eye, right; OS: eye, left; HM: hand movement; FC: finger counting; RAPD: relative afferent pupillary defect; F-VEP: flash visual evoked potential; P-VEP: pattern visual evoked potential; OCT: optical coherence tomography; RNFL: retinal nerve fiber layer; VFD: visual field defect.

protein structural modeling that this mutation leads to the mitochondrial fusion failure, resulting in the RGC death. Intriguingly, Pesch et al. found a different missense mutation at the same site (c.1313A $>$ T, D438V) in two German DOA families [25]. Both mutations significantly impair the GTPase domain and the dynamin-related core region. Understanding how these mutations cause the RGC death may provide valuable information for developing a therapeutic treatment for DOA.
The clinical characterization of the DOA family in this study revealed a remarkable phenotypic heterogeneity. First, the age of onset varied among patients in this family. For example, patients of subfamily A developed DOA in childhood, whereas the disease was diagnosed in adolescence in patients of subfamily B (Figure 1; Table 1). In particular, patient III-10 in subfamily $\mathrm{C}$ was diagnosed at the age of 5 years, while his twin brother III-9 was diagnosed at the age of 13 years (Figure 1; Table 1), suggesting that the age of onset 
TABLE 2: The comparison of BCVA (logMAR) between smokers/alcohol drinkers and nonsmokers/nondrinkers in patients of the DOA family.

\begin{tabular}{lccc}
\hline Patients & Eyes $(n)$ & BCVA (mean \pm SD) & $t$ \\
\hline Smokers & 8 & $1.950 \pm 0.427$ & 2.521 \\
Nonsmokers & 12 & $1.200 \pm 0.761$ & 0.021 \\
\hline Alcohol drinkers & 12 & $1.917 \pm 0.438$ & 4.270 \\
Nonalcoholdrinkers & 8 & $0.875 \pm 0.658$ & $<0.001$ \\
\hline
\end{tabular}

BCVA: best corrected visual acuity; SD: standard deviation.
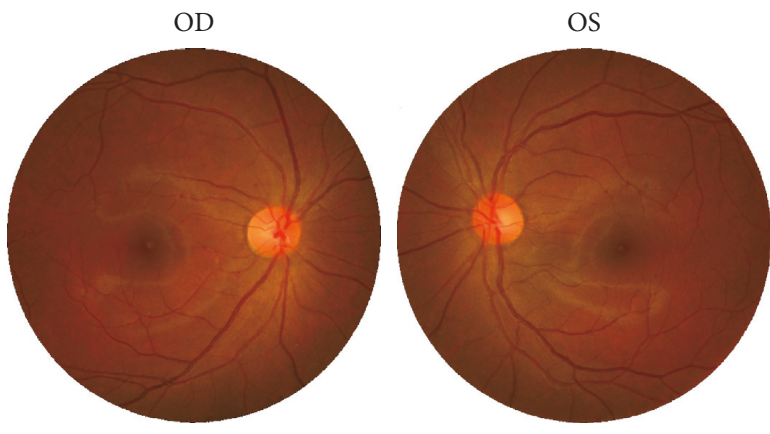

(a)

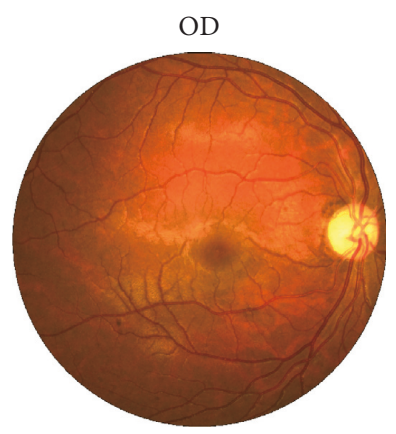

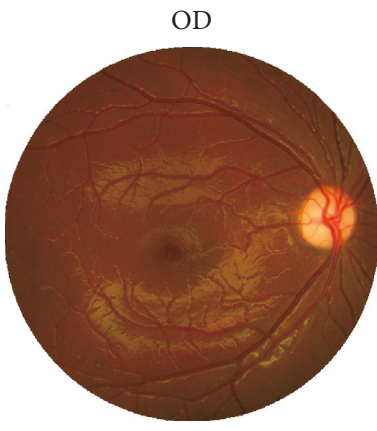

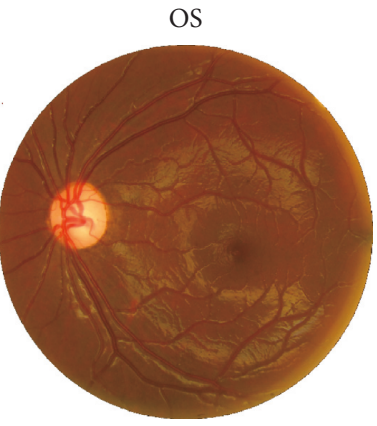

(b)

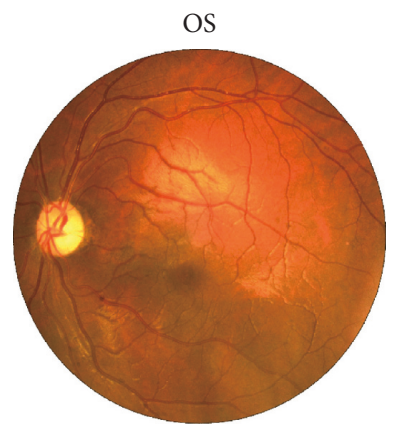

(c)

Figure 2: Fundus photography of three members in the DOA family. The fundus photography of an unaffected family member IV-1 (a) and two DOA patients, V-2 (b) and IV-10 (c). The optic discs of (a) are orange, and their borders are clear. The optic discs of (b) are pale in the temporal area. The optic discs of (c) are pale.

varied by 8 years between the twins. Second, some patients had severely impaired vision of finger counting or hand movement (e.g., III-1, III-3, III-9, III-10, and IV-3), while some other patients had relatively good vision of 20/28 (e.g., IV-12). Third, most of the patients in this family had smoking and/or drinking habits. The smokers/alcoholics had poorer vision, severe optic atrophy, and VEP abnormality (Table 1). Patients who were smokers had significantly lower BCVA than nonsmokers. Similarly, the BCVA of alcohol drinkers was significantly lower than that of nondrinkers in patients (Table 2). Since no matching in gender and age between the groups (smokers vs. nonsmokers and alcohol consumers $v s$. nonalcohol consumers) was possible in this family, these factors might have contributed to the differences in BCVA of the DOA patients. Tobacco and alcohol were well documented as risk factors for toxic and nutritional optic neuropathy, and recent studies had shown that tobacco consumption triggered disease manifestation in LHON [26]. We found tobacco and alcohol consumption to the detriment of DOA, which was in agreement with previous reports that tobacco smoking plays an important role in the pathogenesis of many posterior segment eye diseases $[27,28]$. Since tobacco contains free radical-generating oxidants, the oxidant can cause damage or even death of RGC and loss of retinal nerve fibers through ischemia or high oxidative stress mechanism. Tobacco smoking or chronic nicotine poisoning may cause neurotoxicity of RNFL, resulting in reduced thickness of the retina [28]. Not only smoking can deteriorate optic neuropathy, drinking is also one of the risk factors of optic nerve. Tufan et al. found that chick embryos exposed to different concentrations of ethanol resulted in both retinal degeneration and optic nerve hypoplasia in a dose-dependent manner [29]. Previous studies have also suggested that the combination of two risk factors, smoking and drinking, could contribute to the development of optic neuropathy [30]. Due to the significant heterogeneity in clinical manifestations among patients in the family in this study, we proposed that 

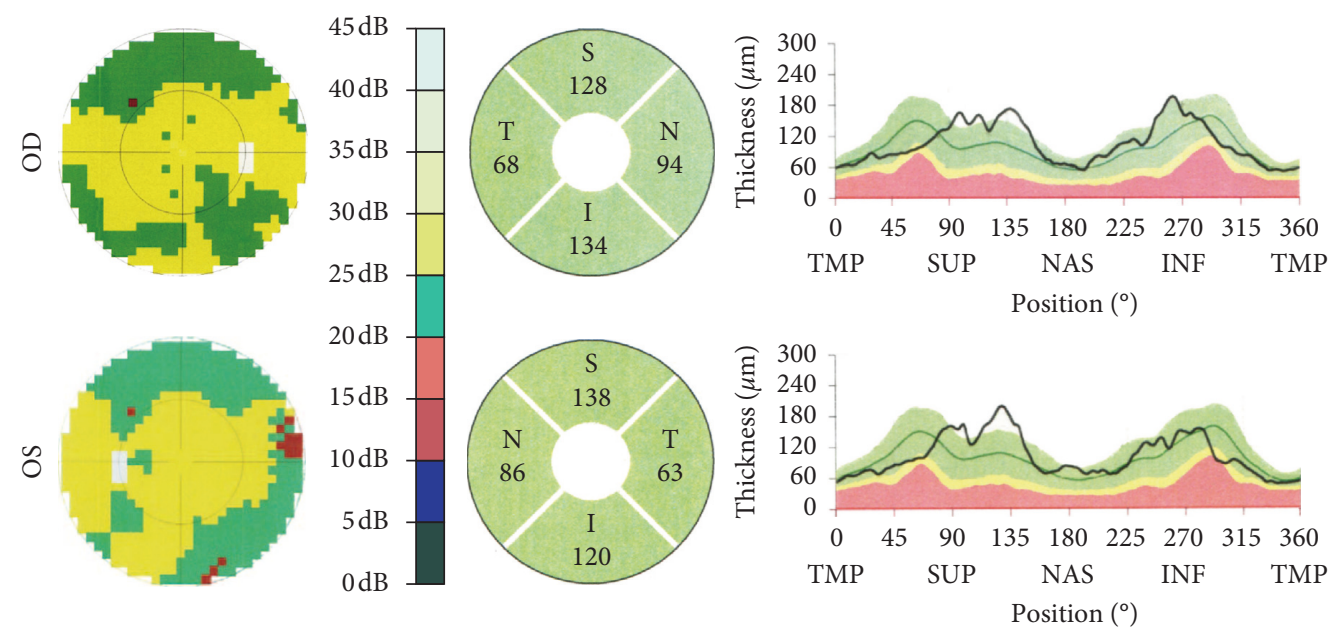

Within normal limits $(P>0.05)$

Borderline $(P<0.05)$

Outside normal limits $(P<0.01)$

(a)
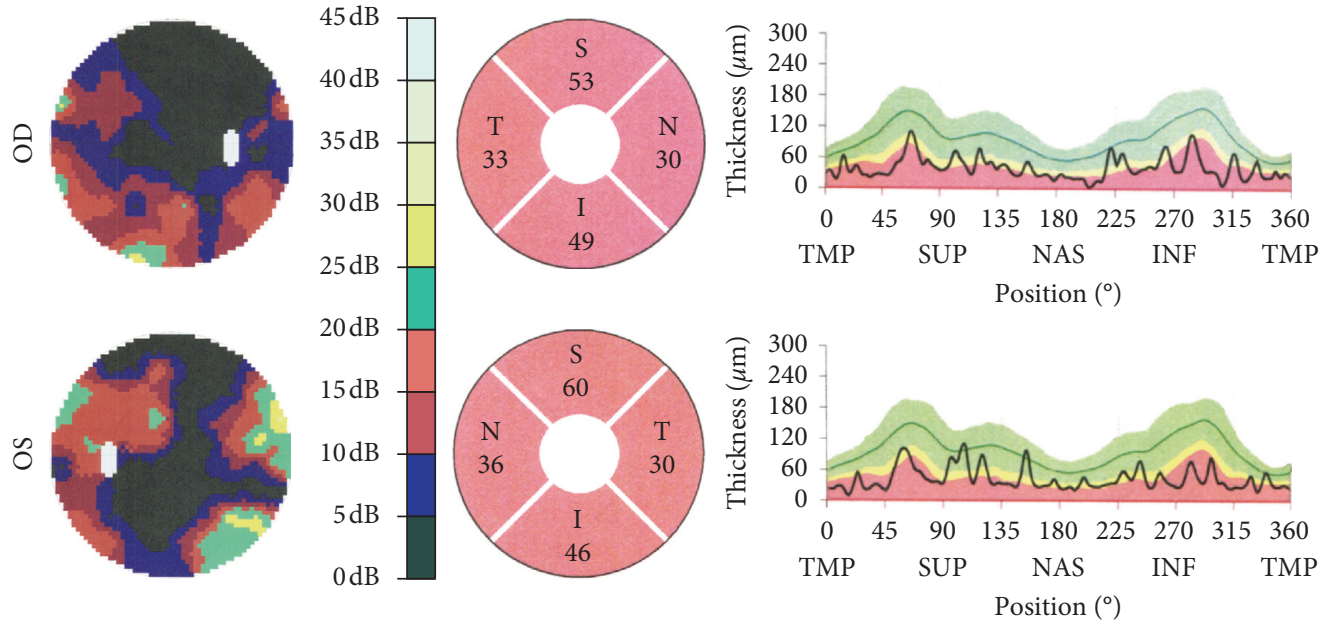

Within normal limits $(P>0.05)$

Borderline $(P<0.05)$

Outside normal limits $(P<0.01)$

(b)

Figure 3: Visual field and OCT exam results of two members in the DOA family. The bilateral visual field (left panel) and OCT exam (middle and right panels) results of an unaffected family member IV-1 (a) and a DOA patient III-9 (b). (a) had normal visual field (dynamic balance test strategy) and normal OCT-RNFL thickness. The range of RNFL thickness for four quadrants was showed in the right panel. (b) had bilateral extensive VFD (central low vision test strategy) and bilateral extensive thinned OCT-RNFL thickness.

environmental factors and living habits such as smoking and drinking could lead to the phenotype heterogeneity of DOA.

In clinics, the diagnosis of DOA needs to be differentiated from LHON, which is also a hereditary optic neuropathy. LHON is more common in young males and may have unilateral progression of vision loss. The fundus exam shows congested or hydropic optic disc borders and dilated peripheral capillaries in the early stage. Pale temporal optic disc and bilateral central or paracentral scotoma may be found in the later stage [31]. No gender difference was indicated in the family in this study (male-to-female ratio of 7 : 5 ), and the symptoms were observed in both eyes. The fundus exam of proband V-2 showed temporal pale optic disc without any congestion and no dilated peripheral capillaries. These clinical features support the initial diagnosis of DOA. Genetic tests can assist in differential diagnosis of DOA from LHON. LHON is a maternally inherited disease due to mitochondrial DNA mutation. The most common mutations are found in the NADH dehydrogenase subunit (ND) 4, ND1, and ND6 genes. Mutations in ND4 m.11778G>A, ND1 m.3460G>A, and ND6 m.14484T>C account for $50 \%-70 \%, 6 \%-25 \%$, and $10 \%-15 \%$ of LHON patients, respectively [32-34]. In this study, we did not find any mtDNA mutations in LHON 


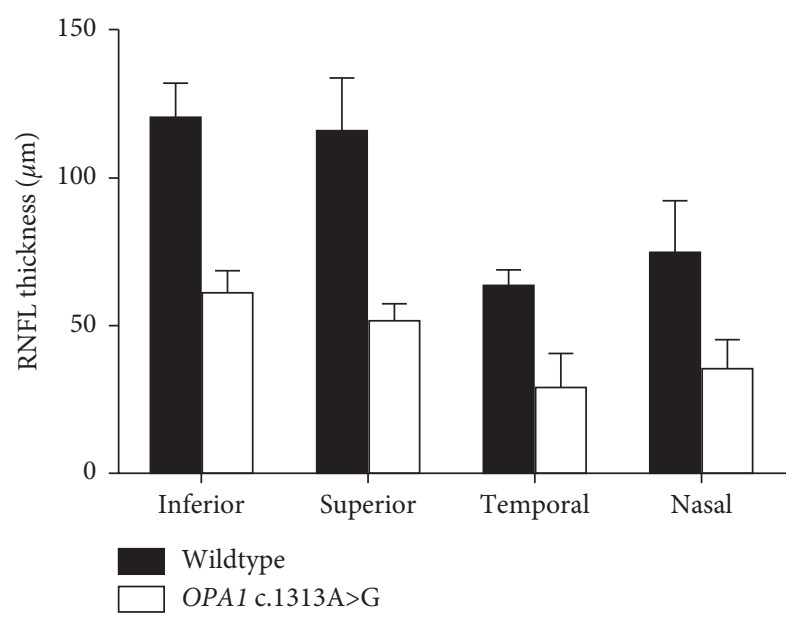

FIgURE 4: The comparison of RNFL thickness between patients and controls in four quadrants in the DOA family. $X$ axis denotes the inferior, superior, temporal, and nasal quadrants. $Y$ axis denotes the RNFL thickness $(\mu \mathrm{m})$. The RNFL thickness of patients was significantly thinner than that of controls, respectively, in inferior, superior, temporal, and nasal quadrants; all $P<0.05(n=16)$.
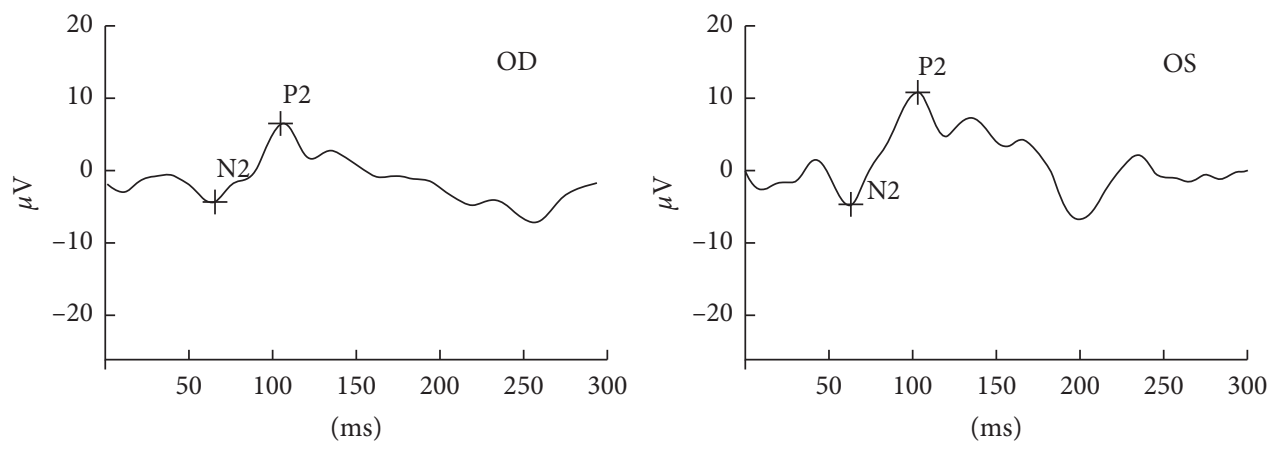

(a)
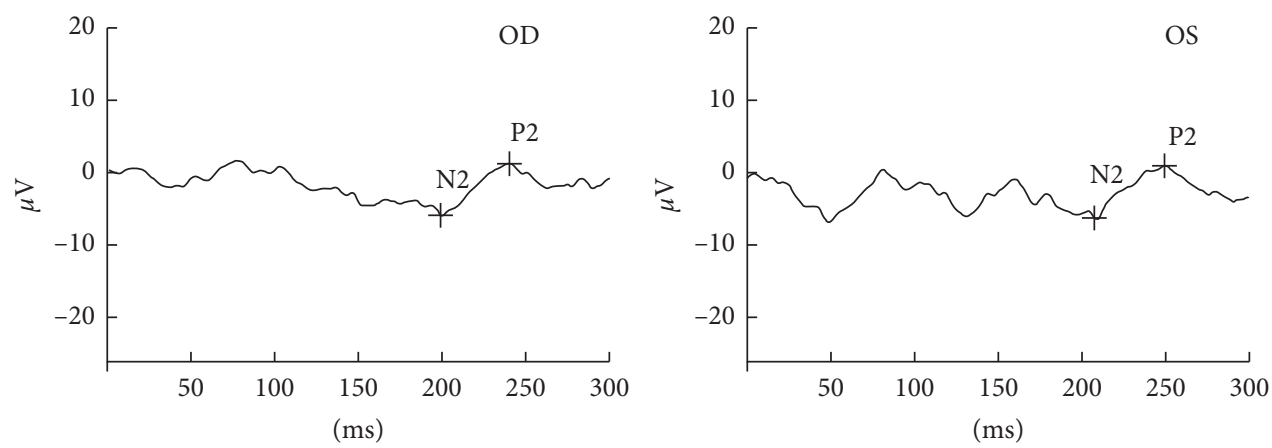

(b)
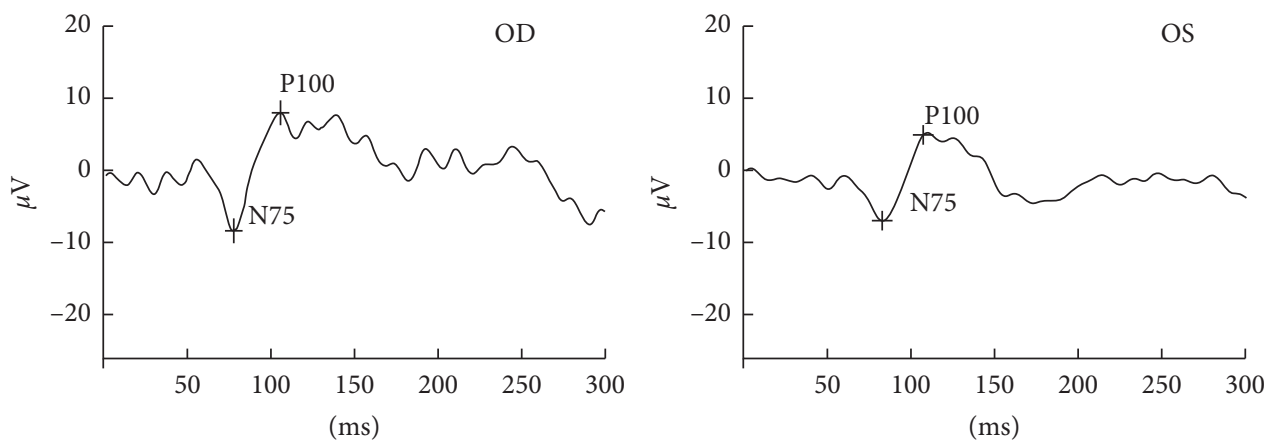

(c)

FIGURE 5: Continued. 

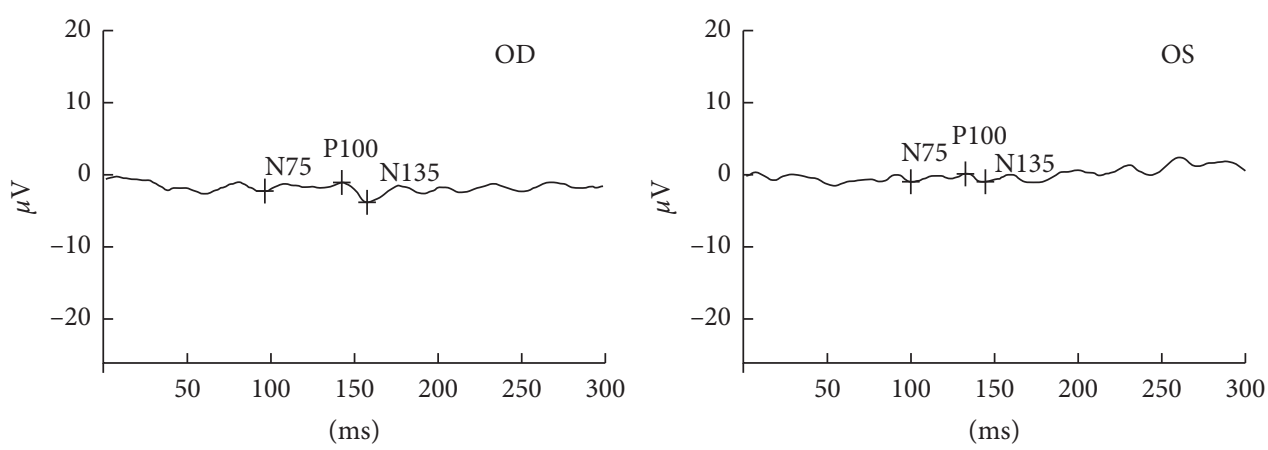

(d)

Figure 5: VEP test results of four members in the DOA family, and F-VEP tests were presented. (a) An unaffected family member III-5 showing a normal bilateral F-VEP pattern. (b) A DOA patient IV-5 showing prolonged P-wave latencies and severe lower P-wave amplitudes in both eyes. (c) P-VEP tests were presented in an unaffected family member IV-1, showing a general normal bilateral P-VEP pattern. (d). A DOA patient IV-3 showing a severe P100 amplitude reduction in both eyes.

TABLE 3: Total number of variants called from the whole exome sequencing data.

\begin{tabular}{lcccccc}
\hline Individual & V-2 & III-3 & III-9 & IV-5 & IV-1 & Total \\
\hline SNPs & 187,398 & 195,216 & 192,720 & 174,243 & 185,388 & 392,379 \\
Indels & 23,794 & 25,222 & 25,231 & 22,832 & 23,801 & 58,595 \\
\hline
\end{tabular}

SNP: single nucleotide polymorphism; indel: insertion/deletion.

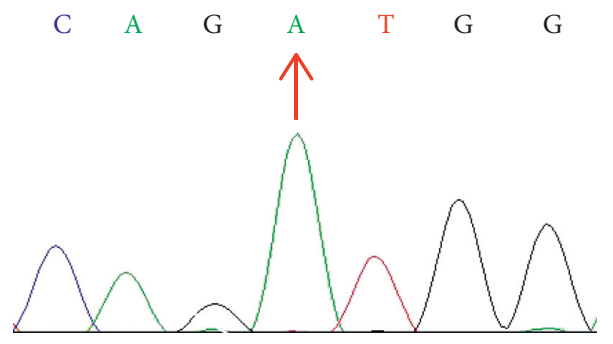

(a)

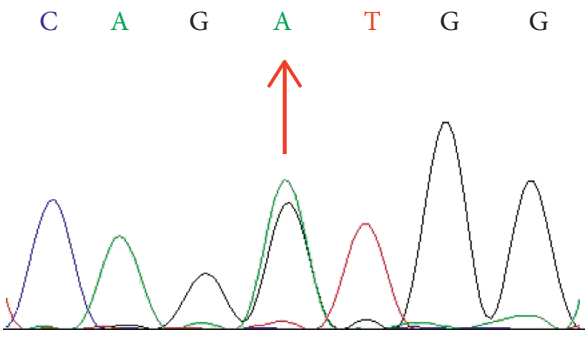

(b)

FIGURE 6: Sanger sequencing chromatogram showing a portion of the sequenced region of OPA1. (a) A wildtype (arrow) of a control. (b) A heterozygous missense mutation (c.1313A $>\mathrm{G}$, arrow) of a patient.

patients. In contrast, DOA is an autosomal inherited optic neuropathy, mainly caused by mutations of the OPA1 gene. To date, five genes (OPA1, OPA3 [35], AFG3L2 [36], DNM1L [37], and WFS1 [38]) have been identified as disease-causing genes for nonsyndromic DOA. In addition, six genetic loci have been reported to be responsible for DOA, including OPA2 (Xp11.4-p11.21), OPA4 (18q12.2-q12.3), OPA5 (22q12.1-q13.1), OPA6 (8q21-q22), OPA7 (11q14.1), and OPA8 (16q21-q22) [2,6]. Among them, OPA1 is the leading gene of DOA [39]. More than 280 OPA1 mutations have been reported to responsible for DOA (http://mitodyn.org; updated on December 30, 2018).

The drug treatment with idebenone and gene therapy such as rAAV2/2-ND4 has been shown to be effective for LHON in clinical trials $[40,41]$. Unfortunately, no clinically proven treatment option for DOA is available yet. Avoiding harmful environmental factors such as tobacco and alcohol exposure is highly recommended to ameliorate the condition. Translational research for DOA is entering an accelerated phase with the availability of animal models, and a variety of pharmacological and genetic therapies are being developed [42, 43]. Further functional studies are needed to provide further insights into this inherited eye disease.

\section{Conclusions}

In summary, we have identified a missense mutation in $O P A 1$ responsible for the disease in a Chinese family affected with DOA. Clinical manifestations of the patients were heterogeneous in this family. This is the first report on the c.1313A $>$ G (p.D438G) mutation of OPA1 identified in a Chinese DOA family.

\section{Data Availability}

The data used to support the findings of this study are included within the article. 


\section{Conflicts of Interest}

The authors declared no conflicts of interest.

\section{Authors' Contributions}

Shaoyi Mei and Xiaosheng Huang contributed equally to this work. Jun Zhao contributed to study concept and design. Jun Zhao, Shaoyi Mei, Xiaosheng Huang, Shiming Peng, Tianhui Zhu, Liang Chen, and Yan Wang performed acquisition of clinical and experimental data with their professional affiliation. Shaoyi Mei and Lin Cheng were involved in statistical analysis, figure making, and article writing. Jun Zhao and Shaoyi Mei performed critical revision of the manuscript for important intellectual content and language editing.

\section{Acknowledgments}

This work was supported by the Science, Technology, and Innovation Commission of Shenzhen Municipality (grant no. GJHZ20180420180937076) and Sanming Project of Medicine in Shenzhen (grant no. SZSM201812090). The authors thank all the family members participated in the study.

\section{References}

[1] P. Kjer, "Infantile optic atrophy with dominant mode of inheritance: a clinical and genetic study of 19 Danish families," Acta Ophthalmologica, vol. 164, no. 54, pp. 1-147, 1959.

[2] B. J. Fan, "Inherited optic neuropathies: genetics and new directions for diagnosis and therapy," CML-Ophthalmology, vol. 24, no. 3, pp. 73-80, 2014.

[3] P. Yu-Wai-Man, P. G. Griffiths, A. Burke et al., "The prevalence and natural history of dominant optic atrophy due to OPA1 mutations," Ophthalmology, vol. 117, no. 8, pp. 1538-1546, 2010.

[4] A. C. Cohn, C. Toomes, C. Potter et al., "Autosomal dominant optic atrophy: penetrance and expressivity in patients with OPA1 mutations," American Journal of Ophthalmology, vol. 143, no. 4, pp. 656-662, 2007.

[5] P. Yu-Wai-Man, P. G. Griffiths, and P. F. Chinnery, "Mitochondrial optic neuropathies - disease mechanisms and therapeutic strategies," Progress in Retinal and Eye Research, vol. 30, no. 2, pp. 81-114, 2011.

[6] G. Lenaers, C. P. Hamel, C. Delettre et al., "Dominant optic atrophy," Orphanet Journal of Rare Diseases, vol. 7, no. 1, p. 46, 2012.

[7] P. M. Skidd, S. Lessell, and D. M. Cestari, "Autosomal dominant hereditary optic neuropathy (ADOA): a review of the genetics and clinical manifestations of ADOA and ADOA+," Seminars in Ophthalmology, vol. 28, no. 5-6, pp. 422-426, 2013.

[8] D. S. Lynch, S. H. Y. Loh, J. Harley et al., "Nonsyndromic Parkinson disease in a family with autosomal dominant optic atrophy due to OPA1 mutations," Neurology Genetics, vol. 3, no. 5, p. e188, 2017.

[9] C. Alexander, M. Votruba, U. E. A. Pesch et al., "OPA1, encoding a dynamin-related GTPase, is mutated in autosomal dominant optic atrophy linked to chromosome 3q28," Nature Genetics, vol. 26, no. 2, pp. 211-215, 2000.
[10] C. Delettre, G. Lenaers, J.-M. Griffoin et al., "Nuclear gene $O P A 1$, encoding a mitochondrial dynamin-related protein, is mutated in dominant optic atrophy," Nature Genetics, vol. 26, no. 2, pp. 207-210, 2000.

[11] Y. Li, J. Li, X. Jia, X. Xiao, S. Li, and X. Guo, "Genetic and clinical analyses of DOA and LHON in 304 Chinese patients with suspected childhood-onset hereditary optic neuropathy," PLoS One, vol. 12, no. 1, Article ID e0170090, 2017.

[12] M. Inoue, N. Himori, H. Kunikata et al., "The reduction of temporal optic nerve head microcirculation in autosomal dominant optic atrophy," Acta Ophthalmologica, vol. 94, no. 7, pp. e580-e585, 2016.

[13] D. Pierron, M. Ferré, C. Rocher et al., "OPA1-related dominant optic atrophy is not strongly influenced by mitochondrial DNA background," BMC Medical Genetics, vol. 10, p. 70, 2009.

[14] P. F. Cherukuri, V. Maduro, K. V. Fuentes-Fajardo et al., "Replicate exome-sequencing in a multiple-generation family: improved interpretation of next-generation sequencing data," BMC Genomics, vol. 16, p. 998, 2015.

[15] H. Li and R. Durbin, "Fast and accurate short read alignment with Burrows-Wheeler transform," Bioinformatics, vol. 25, no. 14, pp. 1754-1760, 2009.

[16] H. Li, B. Handsaker, A. Wysoker et al., "The sequence alignment/map format and SAMtools," Bioinformatics, vol. 25, no. 16, pp. 2078-2079, 2009.

[17] A. McKenna, M. Hanna, E. Banks et al., "The Genome Analysis Toolkit: a MapReduce framework for analyzing nextgeneration DNA sequencing data," Genome Research, vol. 20, no. 9, pp. 1297-1303, 2010.

[18] K. Wang, M. Li, and H. Hakonarson, “ANNOVAR: functional annotation of genetic variants from high-throughput sequencing data," Nucleic Acids Research, vol. 38, no. 16, p. e164, 2010.

[19] K. Schulze-Bonsel, N. Feltgen, H. Burau, L. Hansen, and M. Bach, "Visual acuities "hand motion" and "counting fingers" can be quantified with the Freiburg visual acuity test," Investigative Opthalmology \& Visual Science, vol. 47, no. 3, pp. 1236-1240, 2006.

[20] C. Cid-Castro, D. R. Hernández-Espinosa, and J. Morán, "ROS as regulators of mitochondrial dynamics in neurons," Cellular and Molecular Neurobiology, vol. 38, no. 5, pp. $995-1007,2018$.

[21] A. M. C. Millet, A. M. Bertholet, M. Daloyau et al., "Loss of functional OPA1 unbalances redox state: implications in dominant optic atrophy pathogenesis," Annals of Clinical and Translational Neurology, vol. 3, no. 6, pp. 408-421, 2016.

[22] M. Norton, A. C.-H. Ng, S. Baird et al., "ROMO1 is an essential redox-dependent regulator of mitochondrial dynamics," Science Signaling, vol. 7, no. 310, p. ra10, 2014.

[23] P. Belenguer and L. Pellegrini, "The dynamin GTPase OPA1: more than mitochondria?," Biochimica et Biophysica Acta (BBA)Molecular Cell Research, vol. 1833, no. 1, pp. 176-183, 2013.

[24] S. Dadgar, O. Hagens, S. R. Dadgar et al., "Structural model of the OPA1 GTPase domain may explain the molecular consequences of a novel mutation in a family with autosomal dominant optic atrophy," Experimental Eye Research, vol. 83, no. 3, pp. 702-706, 2006.

[25] U. E. A. Pesch, B. Leo-Kottler, S. Mayer et al., "OPA1 mutations in patients with autosomal dominant optic atrophy and evidence for semi-dominant inheritance," Human Molecular Genetics, vol. 10, no. 13, pp. 1359-1368, 2001.

[26] L. Giordano, S. Deceglie, P. d'Adamo et al., "Cigarette toxicity triggers Leber's hereditary optic neuropathy by affecting 
mtDNA copy number, oxidative phosphorylation and ROS detoxification pathways," Cell Death \& Disease, vol. 6, no. 12, Article ID e2021, 2015.

[27] M. Nita and A. Grzybowski, "Smoking and eye pathologies: a systemic review. part II. Retina diseases, uveitis, optic neuropathies, thyroid-associated orbitopathy," Current Pharmaceutical Design, vol. 23, no. 4, pp. 639-654, 2017.

[28] M. S. Dervişoğulları, Y. Totan, A. Tenlik, A. Yüce, and E. Güler, "Effect of smoking on retina nerve fiber layer and ganglion cell-inner plexiform layer complex," Cutaneous and Ocular Toxicology, vol. 34, no. 4, pp. 282-285, 2015.

[29] A. C. Tufan, G. Abban, I. Akdogan, D. Erdogan, and C. Ozogul, "The effect of in ovo ethanol exposure on retina and optic nerve in a chick embryo model system," Reproductive Toxicology, vol. 23, no. 1, pp. 75-82, 2007.

[30] A. Grzybowski, M. Zülsdorff, H. Wilhelm, and F. Tonagel, "Toxic optic neuropathies: an updated review," Acta Ophthalmologica, vol. 93, no. 5, pp. 402-410, 2015.

[31] J. B. Kerrison, "Hereditary optic neuropathies," Ophthalmology Clinics of North America, vol. 14, no. 1, pp. 99-107, 2001.

[32] P. Jiang, M. Liang, J. Zhang et al., "Prevalence of MitochondrialND4Mutations in 1281 han Chinese subjects with Leber's hereditary optic neuropathy," Investigative Opthalmology \& Visual Science, vol. 56, no. 8, pp. 4778-4788, 2015.

[33] M. L. Valentino, P. Barboni, A. Ghelli et al., "The ND1 gene of complex I is a mutational hot spot for Leber's hereditary optic neuropathy," Annals of Neurology, vol. 56, no. 5, pp. 631-641, 2004.

[34] P. F. Chinnery, D. T. Brown, R. M. Andrews et al., "The mitochondrial ND6 gene is a hot spot for mutations that cause Leber's hereditary optic neuropathy," Brain, vol. 124, no. 1, pp. 209-218, 2001.

[35] P. Reynier, P. Amati-Bonneau, C. Verny et al., "OPA3 gene mutations responsible for autosomal dominant optic atrophy and cataract," Journal of Medical Genetics, vol. 41, no. 9, p. e110, 2004.

[36] M. Charif, A. Roubertie, S. Salime et al., "A novel mutation of AFG3L2 might cause dominant optic atrophy in patients with mild intellectual disability," Frontiers in Genetics, vol. 6, p. 311, 2015.

[37] S. Gerber, M. Charif, A. Chevrollier et al., "Mutations in $D N M 1 L$, as in OPA1, result in dominant optic atrophy despite opposite effects on mitochondrial fusion and fission," Brain, vol. 140, no. 10, pp. 2586-2596, 2017.

[38] B. F. Hogewind, R. J. Pennings, F. A. Hol et al., "Autosomal dominant optic neuropathy and sensorineual hearing loss associated with a novel mutation of WFS1," Molecular Vision, vol. 16, pp. 26-35, 2010.

[39] E. D. Gaier, K. Boudreault, I. Nakata et al., "Diagnostic genetic testing for patients with bilateral optic neuropathy and comparison of clinical features according to OPA1 mutation status," Molecular Vision, vol. 23, pp. 548-560, 2017.

[40] N. Gueven, "Idebenone for Leber's hereditary optic neuropathy," Drugs of Today, vol. 52, no. 3, pp. 173-181, 2016.

[41] C. Vignal, S. Uretsky, S. Fitoussi et al., "Safety of rAAV2/2ND4 gene therapy for Leber hereditary optic neuropathy," Ophthalmology, vol. 125, no. 6, pp. 945-947, 2018.

[42] A. H. Manickam, M. J. Michael, and S. Ramasamy, "Mitochondrial genetics and therapeutic overview of Leber's hereditary optic neuropathy," Indian Journal of Ophthalmology, vol. 65, no. 11, pp. 1087-1092, 2017.

[43] B. Y. Chun and J. F. Rizzo III, "Dominant optic atrophy and Leber's hereditary optic neuropathy: update on clinical features and current therapeutic approaches," Seminars in $\mathrm{Pe}$ diatric Neurology, vol. 24, no. 2, pp. 129-134, 2017. 


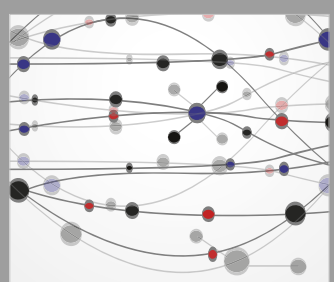

The Scientific World Journal
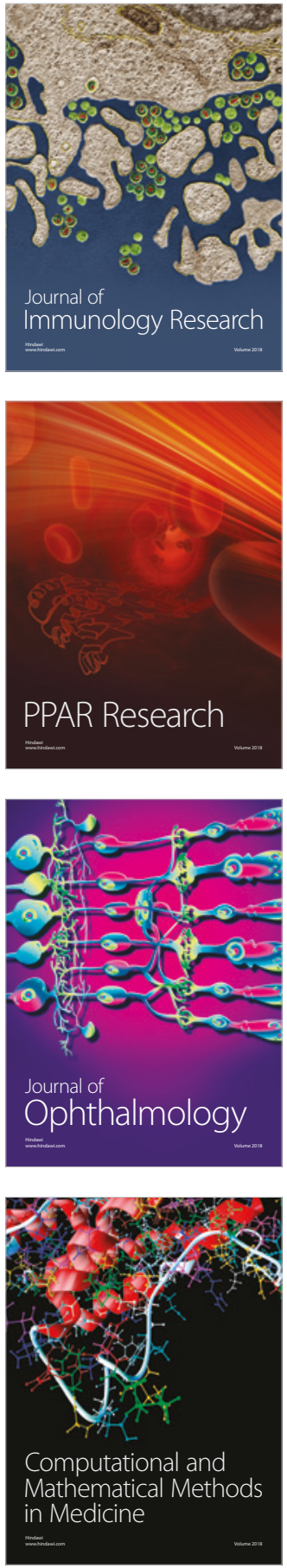

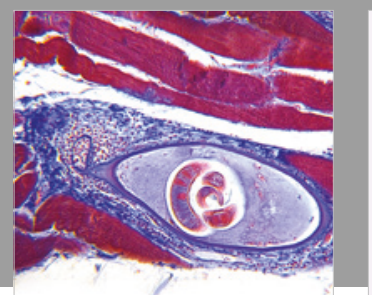

Gastroenterology Research and Practice

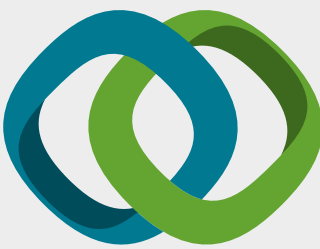

\section{Hindawi}

Submit your manuscripts at

www.hindawi.com
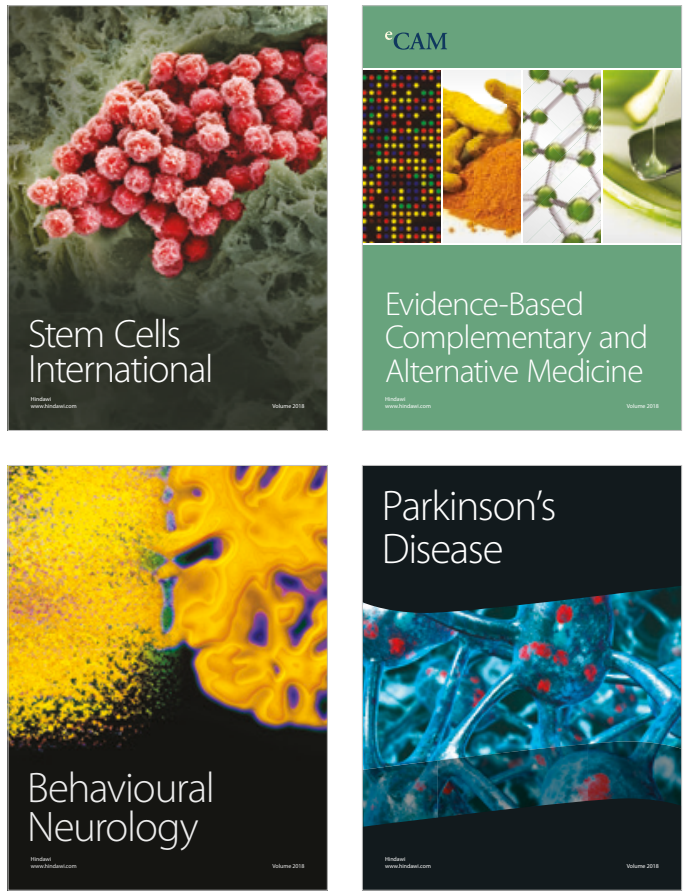

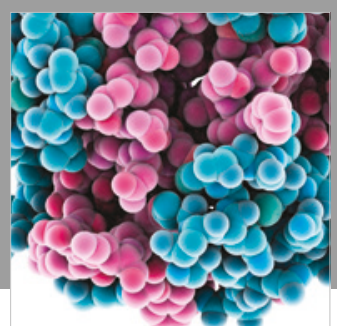

ournal of

Diabetes Research

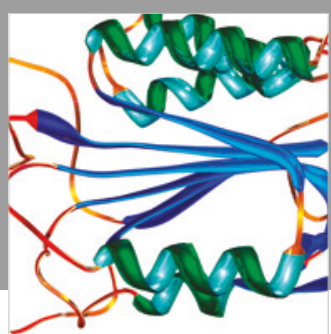

Disease Markers
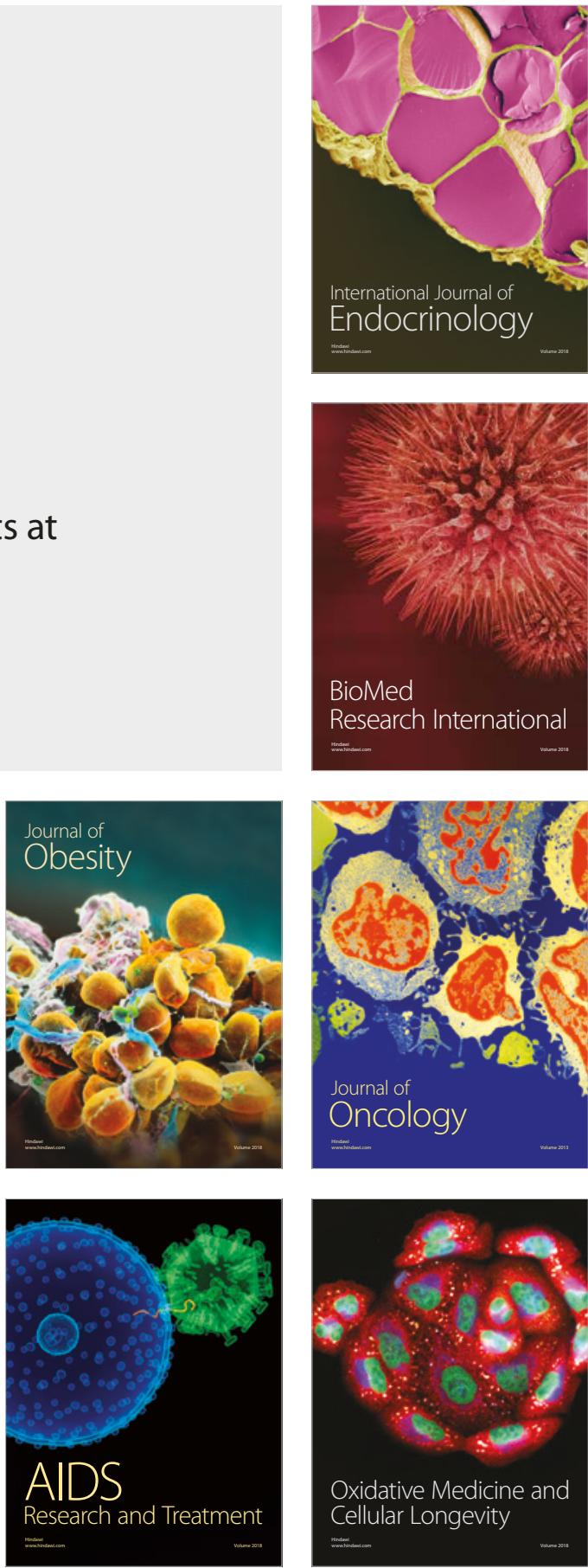